\title{
Effect of dietary blueberry (Vaccinium ashei Reade) leaves on serum and hepatic lipid levels in rats
}

\author{
Kumiko Yuji ${ }^{1,2}$, Hiroshi Sakaida ${ }^{3}$, Takanori Kai ${ }^{3}$, Nobuhiro Fukuda ${ }^{4}$, Chizuko Yukizaki ${ }^{5}$, \\ Miho Sakai ${ }^{5}$, Hirohito Tsubouchi ${ }^{6}$ and Hiroaki Kataoka ${ }^{2}$ \\ ${ }^{1}$ Miyazaki Prefectural Industrial Support Foundation, Miyazaki, Japan, \\ ${ }^{2}$ Section of Oncopathology and Regenerative Biology, Faculty of Medicine, University of Miyazaki, Miyazaki, Japan, \\ ${ }^{3}$ Unkai Shuzo Co., Ltd., Miyazaki, Japan, \\ ${ }^{4}$ Professor emeritus, Faculty of Agriculture, University of Miyazaki, \\ ${ }^{5}$ Miyazaki Prefectural Food R\&D Center, Miyazaki, Japan, and \\ ${ }^{6}$ Digestive Disease and Lifestyle-related Disease Health Research, Human and Environmental Sciences, Kagoshima University Graduate \\ School of Medicine and Dental Sciences, Kagoshima, Japan
}

\begin{abstract}
The serum and liver lipid-lowering effects of dietary freeze-dried blueberry leaf powder (BL) and its hydrothermal extract (BLHE) were examined in rats fed diets with or without cholesterol supplementation. Administration of $1 \%$ and $3 \%$ BL had no adverse effects on food intake or growth; however, relative liver weights were reduced in rats fed diets with and without dietary cholesterol. In the absence of dietary cholesterol, a dose-dependent reduction was evident. The effects of dietary BL on the concentration of serum lipids were marginal; however, the effects on liver triacylglycerol (TG) and cholesterol levels were apparently dose-dependent when the animals were fed diets free of cholesterol. Further, BL significantly attenuated dietary cholesterol-dependent accumulation of hepatic cholesterol, but not of TG. Hydrothermal treatment studies suggested that the active component of BL in terms of its liver lipid-lowering activity is relatively stable at high temperatures. Histopathological analysis of hepatic tissues revealed that BL administration suppresses fatty infiltrations induced by an AIN 76-based high-sucrose diet. The results of this study suggest that some of the active components of BL extracts, which are incorporated into the liver, prevent fatty liver in rats. These results provide further support for the investigation of dietary BL and derivatives thereof as functional human foods.
\end{abstract}

Key words: blueberry leaf, Vaccinium ashei Reade, lipid-lowering activity, fatty liver

\section{INTRODUCTION}

Metabolic syndrome has been recently claimed to be a major public health concern in western countries, including Japan $^{1)}$. This syndrome is characterized by so-called lifestyle-related diseases such as diabetes, obesity, hypertension, and dyslipidemia, which are, in turn, thought to contribute to a drastic elevation in the risk of cardiovascular diseases ${ }^{1-3)}$. On the other hand, recent epidemiological surveys have suggested the potential usefulness of alternative therapies, including herbs and/or Chinese medicines, to prevent some of these lifestyle-related diseases ${ }^{4-7)}$. However, scientific evidence supporting these benefits is still required. In addition, numerous naturally derived agricultural products have been recently shown to contain various types of active components such as plant sterols, flavonoids, terpenoids, alkaloids, and phenolics, among other substances $^{8-11)}$. In addition, various herbs and Chinese medicines may have the potential to prevent lifestyle-related diseases.

In a project to identify effective agricultural products that could cure or reduce the risk of progression of disease caused by hepatitis $\mathrm{C}$ virus (HCV), we speculated that agricultural products may suppress the development of this disease and screened agricultural products (1700 samples from 283 species) indigenous to Miyazaki for their suppressive activity. Blueberry leaves were found to have a notable suppressive potential ${ }^{12)}$. In parallel with this project, we also examined the preventive effects of blueberry leaf extract on lifestyle-related diseases such as lipid disorders. We used an animal model that can be used under various nutritional conditions, because blueberry extract has been shown to have functional activity such as antihyperten-

\footnotetext{
*Correspondence to: Nobuhiro Fukuda, Present address, Imagawa 2-5-23-601, Chuou-ku, Fukuoka City, Fukuoka 810-0054, JAPAN

E-mail: nfukuda@cc.miyazaki-u.ac.jp

Accepted September 4, 2012 (recieved for review May 8, 2012)

Journal of Oleo Science ISSN 1345-8957 print / ISSN 1347-3352 online

http://www.jstage.jst.go.jp/browse/jos/ http://mc.manusriptcentral.com/jjocs
} 
sive $^{13,14)}$, antiatherosclerotic ${ }^{15)}$, and antidiabetic activities ${ }^{16-18)}$. In particular, the blueberry (Vaccinium species) leaf has shown to have hypoglycemic potential in diabetic rats ${ }^{16)}$ as well as in human subjects with type II diabetes ${ }^{18)}$. Thus, we investigated the influence of dietary blueberry leaf and its hydrothermal extract on lipid metabolism in the rat, and report our findings herein.

\section{EXPERIMENTAL PROCEDURES}

\subsection{Plant materials and extraction procedure}

Blueberry (Vaccinium ashei Reade; Rabbiteye, Homebell) plants were cultivated by a local farmer(Unkai farm Co., Miyazaki, Japan), and their leaves were cut into small pieces, freeze dried, and powdered. This preparation is hereafter referred to as "BL." To prepare the hydrothermal extract, $\mathrm{BL}(1 \mathrm{~g} / 30 \mathrm{~mL})$ was mixed with water at $95^{\circ} \mathrm{C}$ for 30 min with continuous stirring, followed by centrifugation; the supernatant thus obtained was again freeze dried and powdered. The resultant preparation is hereafter referred to as "BLHE."

\subsection{Animals and diets}

Male Sprague-Dawley rats weighing 90-110 g (5-wkold) in experiment $1,90-100 \mathrm{~g}(5 \mathrm{wk}$ old $)$ in experiment 2 , and $80-100 \mathrm{~g}(4-5 \mathrm{wk}$ old $)$ in experiment 3 were obtained from Kyudo (Kumamoto, Japan). Rats were fed a commercial powdered stock chow (Type CE-2, Clea Japan, Tokyo, Japan) and acclimatized for 1 week in a temperature-controlled room (between $22^{\circ} \mathrm{C}$ and $24^{\circ} \mathrm{C}$; light on from $0800 \mathrm{~h}$ to $2000 \mathrm{~h}$ ). The rats were then divided into 3 groups with 7 or 8 animals in each group (experiment 1), 4 groups with 5 animals in each group (experiment 2), and 3 groups with 9 animals in each group (experiment 3 ). The rats had free access to control or experimental diets for 30 days in experiments 1 and 2 and for 14 days in experiment 3. The control diet was prepared according to the recommendations of the American Institute of Nutrition ${ }^{19)}$ and contained ( $\%$ by weight) casein, 20\%; corn starch, 15\%; corn oil, 5\%; cellulose, 5\%; mineral mixture (AIN 76) , 3.5\%; vitamin mixture (AIN 76) ,1.0\%; DL-methionine, $0.3 \%$; choline bitartrate, $0.2 \%$; and sucrose to $100 \%$. In experiment 1 , the concentration of sucrose was reduced to accommodate the incorporation of $1 \%$ or $3 \%$ BL as required. In experiment $2, \mathrm{BL}(3 \%)$ was added in the presence and absence of cholesterol ( $0.5 \%)$ plus sodium cholate $(0.15 \%)$ at the expense of sucrose as compared to the control diet. In experiment 3, BL was added at the same level (3\%) used in experiment 2 . In addition, another group of rats was fed the control diet and was subsequently administered oral BLHE emulsion ( $3 \mathrm{~mL}$ of $100 \mathrm{mg}$ BLHE/mL water) every day between $0900 \mathrm{~h}$ and $1100 \mathrm{~h}$; this amount was equivalent to approximately one-half of that fed to the BL group.
The control rats received the same amount of water $(3 \mathrm{~mL})$ during experimental administrations. Food intake and body weight were recorded every other day after the commencement of each trial. Rats were killed by withdrawing blood from the portal vein under mild diethyl ether anesthesia, and their livers were immediately excised, rinsed in saline, dried on filter paper, and weighed after the removal of extrahepatic tissues. The livers were then stored at $-80^{\circ} \mathrm{C}$ until analyses.

All experimental protocols and procedures were approved by the Animal Care and Use Committee of the University of Miyazaki, Japan.

\subsection{Analytical procedures}

Serum triacylglycerol (TG), total cholesterol, and HDLcholesterol levels were determined at SRL Inc., Tokyo, Japan. Liver lipids were extracted and purified according to the methods described by Folch et al. ${ }^{20)}$, and TG and cholesterol levels were measured according to previously described methods ${ }^{21)}$. Cholesteryl ester levels were calculated as the difference between total and free cholesterol levels. The atherogenic index was calculated using the following formula: (Total cholesterol-HDL-cholesterol)/ HDL-cholesterol. For histopathological analyses, a section of the liver tissue from each rat was fixed in 10\% formalin and embedded in paraffin. Sections were cut at a thickness of $4 \mu \mathrm{m}$ and stained with hematoxylin and eosin (HE). Lobular inflammation was judged by focal accumulation of inflammatory cells consisting of neutrophils, lymphocytes, and/or macrophages in the hepatic lobule.

\subsection{Statistics}

The data are presented as mean $\pm \mathrm{SE}$. Statistical evaluation of the results was carried out by one-way analysis of variance, followed by multiple comparisons using the Tukey-Kramer test (StatView software, SAS Institute, Cary, $\mathrm{NC}$, USA) or analysis of variance of two-way layout without repetition where appropriate.

\section{RESULTS}

\subsection{Growth parameters}

The effects of dietary BL and its hydrothermal extract (BLHE) on food intake, weight gain, and relative liver weight are shown in Tables 1, 2, and 3, respectively. No significant effects were evident in food intake and growth of rats fed diets supplemented with $\mathrm{BL}(1 \%$ and $3 \%)$ as compared to those without BL, respectively. However, relative liver weights in rats fed diets without cholesterol administration tended to be decreased in rats fed BL. The reduction was significant and dose-dependent after increasing the dietary levels of BL to $3 \%$ in the experimental group (experiment 1), as compared to the correspond- 
Table 1 Effects of dietary blueberry leaf-dried powder (BL) on food intake, body weight, liver weights, and lipid parameters of serum and liver in rats (Experiment 1).

\begin{tabular}{|c|c|c|c|c|c|c|c|c|c|}
\hline & & \multicolumn{2}{|c|}{ Body weight } & \multirow[t]{2}{*}{ Food intake } & \multirow[t]{2}{*}{ Liver weight } & \multicolumn{2}{|c|}{ Serum lipids } & \multicolumn{2}{|c|}{ Liver lipids } \\
\hline & & Initial & Final & & & Triacylglycerol & Cholesterol & Triacylglycerol & Cholesterol \\
\hline & & (g) & (g) & (g/day) & (g/100g BW) & \multicolumn{2}{|c|}{$(\mathrm{mg} / \mathrm{dl})$} & \multicolumn{2}{|c|}{$(\mathrm{mg} / \mathrm{g})$} \\
\hline Control & & $170 \pm 3$ & $427 \pm 6$ & $27.1 \pm 0.5$ & $5.30 \pm 0.11^{\mathrm{a}}$ & $341 \pm 34$ & $118 \pm 6^{a}$ & $79.9 \pm 9.2^{\mathrm{a}}$ & $3.81 \pm 0.26^{\mathrm{a}}$ \\
\hline \multirow{2}{*}{$\mathrm{BL}$} & $1 \%$ & $170 \pm 3$ & $426 \pm 10$ & $27.4 \pm 0.7$ & $4.93 \pm 0.09^{b}$ & $401 \pm 66$ & $109 \pm 8^{\mathrm{ab}}$ & $71.9 \pm 10.1^{\mathrm{a}}$ & $3.55 \pm 0.20^{\mathrm{ab}}$ \\
\hline & $3 \%$ & $169 \pm 3$ & $410 \pm 7$ & $26.7 \pm 0.5$ & $4.49 \pm 0.09^{c}$ & $375 \pm 42$ & $94 \pm 4^{\mathrm{b}}$ & $42.5 \pm 2.7^{\mathrm{b}}$ & $3.01 \pm 0.13^{\mathrm{b}}$ \\
\hline
\end{tabular}

The values are means SE for 7 to 8 rats per group.

${ }^{\text {abc }}$ Values not sharing common superscript letters in the same column are significantly different at $p<0.05$.

Table 2 Effects of blueberry freeze dried powder (BL) on food intake, growth parameters, and lipid parameters of serum and liver in rats fed cholesterol-free and cholesterol-enriched diets (Experiment 2).

\begin{tabular}{|c|c|c|c|c|c|c|c|}
\hline & \multicolumn{2}{|c|}{ Cholesterol-free } & \multicolumn{2}{|c|}{ Cholesterol-enriched } & \multicolumn{2}{|c|}{ ANOVA } & \multirow[b]{2}{*}{ Interaction } \\
\hline & Control & BL & Control & BL & Cholesterol & BL & \\
\hline \multicolumn{8}{|l|}{ Growth parameters } \\
\hline Initial body weight (g) & $165 \pm 3$ & $166 \pm 3$ & $165 \pm 4$ & $165 \pm 2$ & NS & NS & NS \\
\hline Final body weight (g) & $406 \pm 5$ & $398 \pm 5$ & $416 \pm 5$ & $402 \pm 2$ & NS & NS & NS \\
\hline Food intake (g/day) & $24.2 \pm 0.5$ & $24.1 \pm 0.4$ & $24.5 \pm 0.7$ & $23.5 \pm 0.4$ & NS & NS & NS \\
\hline Relative liver (g/100g BW) & $5.17 \pm 0.08$ & $4.50 \pm 0.12$ & $6.48 \pm 0.14$ & $5.65 \pm 0.14$ & $p<0.05$ & $p<0.05$ & NS \\
\hline \multicolumn{8}{|l|}{ Serum parameters (mg/dl) } \\
\hline Triacylglycerol & $431 \pm 50$ & $425 \pm 33$ & $275 \pm 16$ & $304 \pm 40$ & $p<0.05$ & NS & NS \\
\hline \multicolumn{8}{|l|}{ Cholesterol } \\
\hline Total & $112 \pm 7$ & $103 \pm 8$ & $192 \pm 19$ & $158 \pm 18$ & $p<0.05$ & NS & NS \\
\hline HDL & $39.4 \pm 1.4$ & $38.8 \pm 2.0$ & $32.6 \pm 1.1$ & $33.2 \pm 0.9$ & $p<0.05$ & NS & NS \\
\hline Atherogenic index & $1.83 \pm 0.09$ & $1.65 \pm 0.09$ & $4.99 \pm 0.76$ & $3.75 \pm 0.53$ & $p<0.05$ & NS & NS \\
\hline \multicolumn{8}{|l|}{ Liver parameters (mg/g liver) } \\
\hline Triacylglycerol & $50.0 \pm 5.3^{\mathrm{ac}}$ & $26.3 \pm 4.7^{\mathrm{a}}$ & $72.2 \pm 5.3^{\mathrm{bc}}$ & $77.2 \pm 8.0^{\mathrm{b}}$ & $p<0.05$ & NS & $p<0.05$ \\
\hline Cholesterol & $3.24 \pm 0.12^{\mathrm{a}}$ & $2.70 \pm 0.25^{\mathrm{a}}$ & $66.5 \pm 0.6^{b}$ & $57.4 \pm 2.2^{\mathrm{c}}$ & $p<0.05$ & $p<0.05$ & $p<0.05$ \\
\hline
\end{tabular}

The values are means \pm SE for 5 rats per group.

${ }^{\text {abc }}$ Values not sharing common superscript letters in the same row are significantly different at $p<0.05$.

HDL, high density lipoprotein.

Table 3 Effects of dietary blueberry leaf-dried powder (BL) and hydrothermal extracts of BL (BLHE) on food intake, body weight, liver weights, and lipid parameters of serum and liver in rats (Experiment 3).

\begin{tabular}{|c|c|c|c|c|c|c|c|c|}
\hline & \multicolumn{2}{|c|}{ Body weight } & \multirow[t]{2}{*}{ Food intake } & \multirow[t]{2}{*}{ Liver weight } & \multicolumn{2}{|c|}{ Serum lipids } & \multicolumn{2}{|c|}{ Liver lipids } \\
\hline & Initial & Final & & & Triacylglycerol & Cholesterol & Triacylglycerol & Cholesterol \\
\hline & (g) & $(\mathrm{g})$ & (g/day) & $(\mathrm{g} / 100 \mathrm{~g} \mathrm{BW})$ & \multicolumn{2}{|c|}{$(\mathrm{mg} / \mathrm{dl})$} & \multicolumn{2}{|c|}{$(\mathrm{mg} / \mathrm{g})$} \\
\hline Control & $162 \pm 3$ & $275 \pm 4$ & $21.7 \pm 0.3$ & $6.06 \pm 0.21^{\mathrm{a}}$ & $299 \pm 18^{\mathrm{ab}}$ & $115 \pm 7$ & $80.7 \pm 18.8^{a}$ & $4.11 \pm 0.27^{\mathrm{a}}$ \\
\hline $\mathrm{BL}$ & $163 \pm 3$ & $278 \pm 4$ & $22.4 \pm 0.3$ & $5.50 \pm 0.05^{\mathrm{b}}$ & $382 \pm 30^{\mathrm{b}}$ & $100 \pm 4$ & $36.2 \pm 4.3^{\mathrm{b}}$ & $2.99 \pm 0.09^{\mathrm{b}}$ \\
\hline $\begin{array}{l}\text { Hydrothermal } \\
\text { extracts of BL } \\
\text { (BLHE) }\end{array}$ & $162 \pm 3$ & $277 \pm 5$ & $21.9 \pm 0.4$ & $5.75 \pm 0.16^{\mathrm{ab}}$ & $283 \pm 24^{\mathrm{a}}$ & $108 \pm 7$ & $45.0 \pm 6.5^{\mathrm{ab}}$ & $3.10 \pm 0.17^{b}$ \\
\hline
\end{tabular}

The values are means \pm SE for 9 rats per group.

${ }^{\mathrm{ab}}$ Values not sharing common superscript letters in the same column are significantly different at $p<0.05$. 
ing control group. Further, oral administration of BLHE emulsion (3 mL of $100 \mathrm{mg}$ BLHE/mL water) to rats fed a control diet caused no detrimental effect on food intake and growth as compared to those fed a control diet. On the other hand, dietary cholesterol resulted in a significant increase in the liver weight, while supplementation of the high-cholesterol diet with BL was very effective in preventing this increase.

\subsection{Serum and liver lipids}

The effects of dietary BL or of oral administration of BLHE on serum TG levels were not consistent in the absence of dietary cholesterol, as shown in Table 1; however, liver TG levels were markedly reduced with increasing levels of BL in the diet (Table 1). The reduction at the $3 \%$ dietary level was statistically significant, and the extent of the reduction was $46.8 \%$. On the other hand, serum and liver total cholesterol levels also decreased in a dose-dependent manner with increasing dietary levels of BL; the total liver cholesterol-lowering effect at the dietary BL level of 3\% was statistically significant, and the extent of the reduction was $21 \%$. Free-cholesterol levels in the liver remained unchanged, and therefore, the proportion of cholesterol ester was significantly and dose-dependently lower in rats fed high dietary levels of BL.

Administration of cholesterol to the diets free of cholesterol appeared to influence serum lipid levels in a reciprocal manner, as shown in Table 2; thus, dietary cholesterol significantly decreased serum TG and HDL-cholesterol levels, while it increased total cholesterol levels. After alteration in the concentrations of serum total and HDL-cholesterol, the calculated atherogenic index, a known risk factor for coronary heart disease, was significantly elevated in rats fed diets enriched with cholesterol as compared to those fed diets free of cholesterol; however, supplementation of the diets with BL did not affect this index. On the other hand, hepatic TG and cholesterol levels, especially cholesterol levels, were markedly increased after the administration of dietary cholesterol. Dietary BL again induced a $47.4 \%$ reduction in the concentration of liver TGs in the absence of dietary cholesterol, similar to the previously made observation (experiment 1 in Table 1 ). However, the dietary BL-dependent liver-TG lowering effect was absent in rats fed diets enriched with cholesterol. On the other hand, dietary BL tended to decrease liver cholesterol levels; the reduction was significant in the presence of dietary cholesterol.

In experiment 3, we administered BLHE, which was extracted with $\mathrm{BL}$ at $95^{\circ} \mathrm{C}$, and investigated whether this extract is effective in terms of lowering hepatic TG levels. Oral administration of BLHE for 2 wk tended to lower hepatic TG and cholesterol levels; the reduction in the latter lipid component was statistically significant as compared to the controls. Although a direct comparison cannot be made between control and BL-fed rats, dietary BL appears to have hepatic TG- and cholesterol-lowering potential.

\subsection{Histopathological analyses of liver tissues}

Figure 1 shows representative histopathological analyses of liver tissues from various groups of experiments 1,2 , and 3 . It should be noted that the rats exhibited distinct fatty infiltrations, particularly macrovesicular fatty infiltration of the hepatocytes, when they were fed the control diet. However, these infiltrations were apparently abrogated in rats fed diets with 3\% BL (Fig. 1A). In addition, focal inflammatory changes probably caused by steatosis were noted in the livers of control rats, and this change was less evident in livers of BLHE-fed rats (Fig. 1B). On the other hand, in experiment 2 , the hepatocytes of rats fed diets enriched with cholesterol exhibited cytoplasm with clear or ground-glass appearance and mild macrovesicular fatty infiltration (Fig. 1C). Although the cytoplasmic alteration moderately decreased after the rats were fed diets containing BL, this was not a significant histological improvement (Fig. 1C). On the other hand, rats fed the control diet but administered dietary hydrothermally treated BL extract (the BLHE group) also exhibited improved outcomes, e.g., fatty infiltration in the liver (Fig. 1D).

\section{DISCUSSION}

The present study clearly showed that dietary BL causes a dose-dependent reduction in relative liver weights in rats fed diets without cholesterol supplementation. In addition, this effect was reproduced in rats fed diets containing cholesterol; the enlargement of livers caused by dietary cholesterol was significantly reduced in the presence of dietary BL. These observations were not associated with changes in food intake or growth and, therefore they suggest that dietary BL is capable of reducing hepatic weight, probably through a decrease in the concentration of hepatic lipids, as discussed below.

Because little is known about the effects of dietary BL on lipid metabolism, we first aimed to determine the effectiveness of BL in reducing the concentration of serum and liver lipids and the amount of BL required in the diet to achieve a marked change in the concentration of these lipids. The animals were fed diets containing $\mathrm{BL}(1 \%$ and $3 \%$ ) without cholesterol (Table 1). Dietary BL was found to be effective in lowering the serum and liver lipid levels, especially liver TG levels. Cholesterol levels were also reduced in a dose-dependent manner, and the reduction was significant at the $3 \%$ level, as shown in Table 1. Cignarella et al. examined the effects of oral administration of an ethanol extract of blueberry leaves on plasma TG levels in various types of hypertriglyceridemia, induced by ad- 

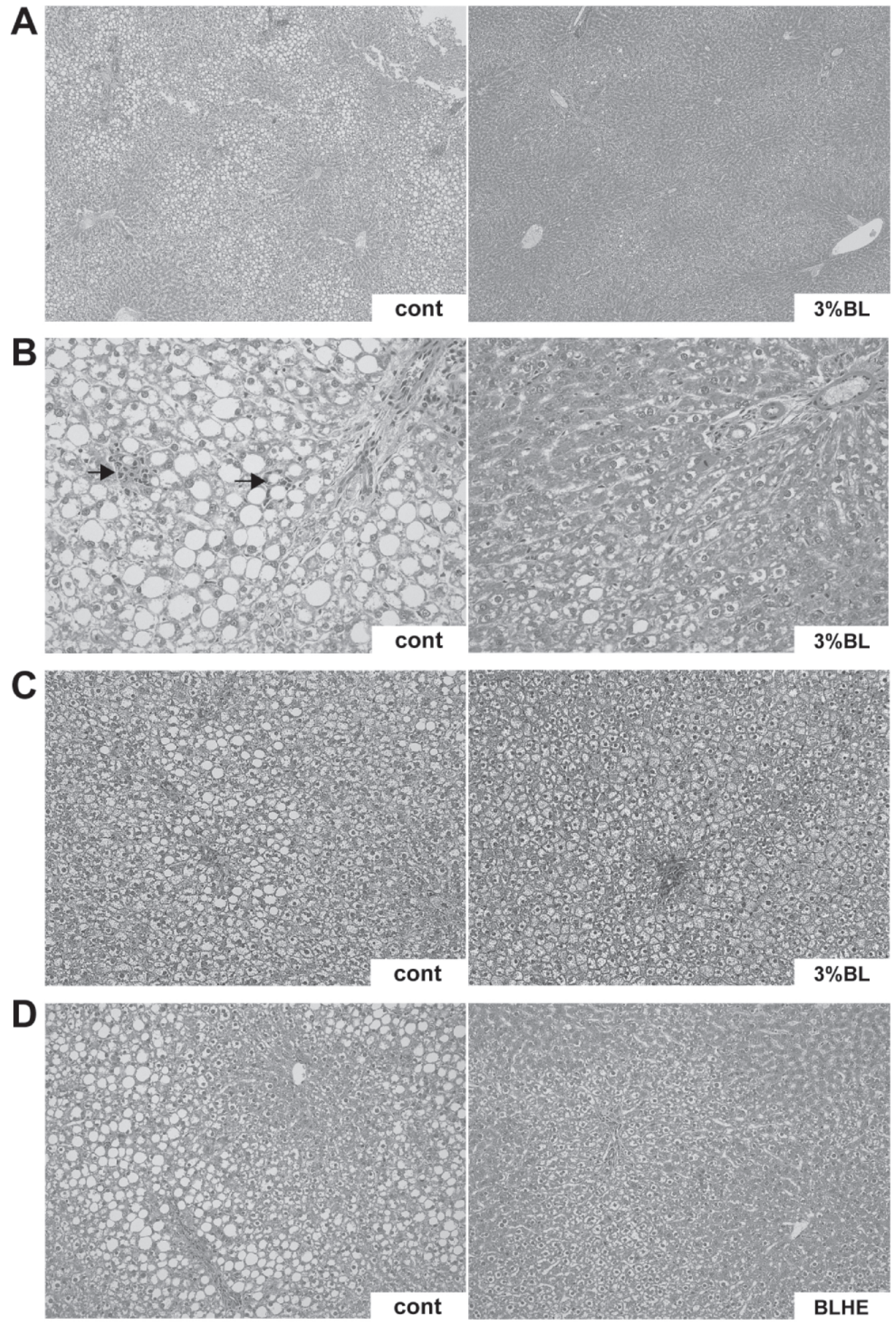

Fig. 1 Histology of the liver tissues (HE stains). (A, B) Liver histology in Experiment 1 showing macrovesicular fatty change of the hepatocytes in control diet rats (cont). Feeding with 3\% BL significantly improved the fatty degeneration. Photos of low magnification image (original magnification, $40 \times)$ and high magnification $(200 \times$ ) are shown in A and B, respectively. Arrows indicate inflammatory reactions. (C) Liver histology of Experiment 2, with enriched cholesterol in diets. (original magnification, $100 \times$ ). (D) Liver histology in Experiment 3. The hydrothermal extract of BL (BLHE) improved liver histology (original magnification, $100 \times$ ). 
ministration of streptozotocin, ethanol treatment, and fructose, as well as in genetically hyperlipidemic Yoshida $\operatorname{rats}^{16)}$. In general, the authors observed a lowering effect by using this extract, but this did not apply to fructose treatment. The blueberry extract failed to prevent the increase in plasma TG levels induced by fructose. Nagao et al. examined the effects of the same freeze-dried blueberry powder preparation used in our current study, at dietary levels of $1 \%$ and $5 \%$, with no cholesterol added to diets, on serum and liver lipid levels in Otsuka Long-Evans Tokushima Fatty (OLETF) rats. They found that the reduction in the concentration of liver lipids (particularly TG) by dietary BL was significantly different at the $5 \%$ level $^{22}$. Using hamsters fed high-fat diets supplemented with blueberry pomace byproducts ( $8 \%$ dried whole blueberry peels, $2 \%$ dried extract of peels, and $6 \%$ residue from extracted peel), Kim et al. showed that all of these preparations significantly lowered total plasma cholesterol and VLDL-cholesterol levels ${ }^{23)}$. Kalt et al. also showed that pigs fed diets supplemented with $1 \%, 2 \%$, and $4 \%$ freeze-dried blueberry leaf powder showed a decrease in total, LDL-, and HDLcholesterol levels, although no dose response was evident ${ }^{24)}$. The antiatherosclerotic effect of dietary freezedried blueberry leaf powder has been shown at the $1 \%$ dietary level in apolipoprotein E-deficient mice, although this effect was independent of an altered serum lipid profile or total antioxidant capacity ${ }^{25)}$. Taken together, the observations made in this study and the observation regarding a potent antihypertensive effect of BL reported by Sakaida et al. ${ }^{13)}$ indicate that BL is a promising candidate for a functional human food. In addition, the level required for the various physiological activities of dietary BL in the absence of dietary cholesterol is less than $3 \%$.

It is well known that dietary cholesterol markedly increases the concentration of serum and liver lipids in experimental animals ${ }^{27,28)}$. As shown in Table 2, although dietary cholesterol significantly elevated both the serum and liver cholesterol levels, it appeared to influence liver TG levels in a reciprocal manner; thus, dietary cholesterol decreased serum TG levels but it caused an increase in the accumulation of liver TG. In addition, there was a marked difference in serum TG levels in the control rats fed diets without added cholesterol in experiment 1 and 2 (Tables 1 and 2). The reason(s) for these observations with regard to the TG levels in the serum and/or liver counterpart are not known at present.

In this study, we examined the influence of dietary BL on serum and liver lipid levels of rats fed diets with and without cholesterol supplementation. We found that dietary BL elicited liver TG-lowering and cholesterol-lowering effects, but had no influence on serum and liver lipid levels in rats fed diets with cholesterol supplementation, except for total liver cholesterol. Total liver cholesterol levels were significantly lower (13.7\%) in rats fed diets sup- plemented with BL than in those fed diets without BL supplementation. The lack of liver TG-lowering activity of BL in rats fed a cholesterol-supplemented diet was attributable to the liver TG-raising effect of dietary cholesterol, which outweighed the TG-lowering activity of dietary BL, suggesting that the lipid-lowering activity of BL is relatively weak.

In experiment 3, we examined the heat stability of active components of $\mathrm{BL}$ via hydrothermal treatment at $95^{\circ} \mathrm{C}$. In this experiment, the animals were administered BLHE orally at approximately half the dose $(300 \mathrm{mg} / 3 \mathrm{~mL})$ used in the BL-ingestion experiments. In terms of liver TG-lowering activity, we found that oral administration of an aqueous extract of hydrothermal BL (BLHE) appeared to correspond to the extent of lipid-lowering activity observed after BL feeding, as shown in Table 1. Inoue et al. ${ }^{28)}$ studied the effects of dietary hot water extract powder prepared from $\mathrm{BL}$ (the same preparation as used in our study) on lipid metabolism in obese OLETF rats. They found that feeding of this powder at the $2 \%$ level resulted in a significant decrease in the concentration of hepatic TG, indicating the usefulness of either administration of drinking or diet for the observed hepatic lipid-lowering effect. In addition, and notably, the hydrothermal stability of BL reported in the current study may prove useful with regard to aspects of food processing such as sterilization, as well as the extraction procedure for decoction of leaves as a folk medicine, the so-called "anti-diabetic teas."

In the current study, we conducted histopathological analyses of fatty infiltration in livers of rats fed diets supplemented with and without cholesterol. We confirmed that dietary BL as well as oral administration of BLHE, as compared to respective control counterparts, are clearly abrogated in fatty livers caused by an AIN 76-based highsucrose diet, but not by dietary cholesterol (Fig. 1).

We are now investigating the potential mechanism(s) and component (s)responsible for the observed reduction in the concentration of liver TGs and cholesterol induced by dietary BL and derivatives thereof. With regard to the physiological relevance of the lipid-lowering activity of dietary BL, Cignarella et al. have shown that the BL administration-dependent reduction in plasma TG levels is attributable to an extrahepatic phenomenon by using Triton WR 1339 treatment. They suggested that BL may stimulate the catabolism of TG-rich lipoproteins, rather than diminish the production of these lipoproteins ${ }^{16)}$. On the other hand, Nagao et al. recently examined the effect of dietary BL on the activities of enzymes related to fatty acid synthesis and fatty acid $\beta$-oxidation in OLETF rats. These authors found that the liver TG-lowering effect of BL might occur via reduced fatty acid synthesis and elevated fatty acid oxidation $^{22)}$. Using the same OLETF rats, Inoue et al. also observed that feeding of the hot water extract of BL (prepared following the same method as that of our BLHE) alle- 
viates hepatic TG accumulation through enhanced lipolysis in the liver ${ }^{28)}$. Thus, these observations suggest that the mechanism (s) responsible for the observed reduction in liver TG levels by dietary BL is, in part, due to both suppressive lipid synthesis and enhanced lipolysis and its oxidation, although clarification of these issues requires further studies.

With regard to the cholesterol-lowering effect of dietary $\mathrm{BL}$ (Tables 1 and 2), Liang et al. recently reported that dietary supplementation of 0.5 and $1 \%$ blueberry anthocyanins for 6 wk lowered plasma and liver cholesterol levels by increasing fecal excretion of acidic and neutral steroids in hamsters fed a high-cholesterol diet. In addition, they demonstrated, by analysis using real-time polymerase chain reaction, that dietary blueberry anthocyanins downregulated the genes of 3-hydroxy-3-methyl-glutaryl-CoA reductase, Niemann-Pick Cl-like protein, AcylCoA:cholesterol acyltransferase-2, microsomal triglyceride transfer protein MTP, and ATP-binding cassette transporter G8, which are known to be major regulatory factors for cholesterol metabolism ${ }^{29)}$. There are no reports, except this one, for the effect of dietary BL on the regulation of the expression of various genes involved in cholesterol metabolism. We are now investigating whether a similar down-regulation of the genes involved in the cholesterol metabolism occurs in rats fed diets containing BL or BLHE.

Polyphenols that occur naturally in the blueberry leaf, e.g., quercetin, caffeic acid, chlorogenic acid, rutin, and procyanidins, have been reported to exhibit various physiological activities, including antioxidant activity, in vitro and in vivo ${ }^{30)}$. In another series of experiments with chlorogenic acid, one of the major polyphenols in $\mathrm{BL}$, we observed no alteration in the concentration of serum and liver lipids when rats were fed diets supplemented with $0.075 \%$ chlorogenic acid, an amount corresponding to that contained in 3\% BL supplementation (data not shown). This finding suggests that chlorogenic acid is not a contributory polyphenol in this context. On the other hand, BL has been reported to contain (in weight \%): carbohydrate, 62.9; protein, 6.2; fat, 5.3; ash, 3.1; moisture, 3.4; and tannin, $18.7^{13)}$. We analyzed dietary fiber (DF) of this sample according to the procedure of Prosky et al. ${ }^{31)}$, since DF has been shown to be active in alleviating lipid disorders, especially hypercholesterolemia, in experimental animals and humans. We found that BL contained 1.7\% water-soluble $\mathrm{DF}$ and $57.9 \%$ water-insoluble DF, accounting for about half of the BL to be insoluble DF. These observations suggest that DF is also a contributing ingredient for the observed reduction in the concentration of liver TGs and cholesterol by dietary BL. We are currently investigating which component(s)are responsible for the liver lipid-lowering activity of BL and related extracts.

In summary, the present results suggest that blueberry leaf has potent liver lipid-lowering activity, especially TG- lowering activity. Therefore, dietary blueberry leaf may be useful for the prevention of fatty liver diseases. Further studies are required to identify the active components and mechanism (s) underlying these beneficial effects of BL and BLHE.

\section{Acknowledgements}

This work was supported by a Grant-in-Aid for Miyazaki Prefecture Collaboration of Regional Entities for the Advancement of Technological Excellence from Japan Science and Technology Agency.

\section{References}

1) Zimmet, P.; Magliano, D.; Matsuzawa, Y.; Albert, G.; Shaw, J. The metabolic syndrome: A global public health problem and a new definition. J. Atheroscler. Thromb. 12, 295-300 (2005).

2) Hegele, R. A.; Pollex, R. L. Genetic and physiological insights into the metabolic syndrome. Am. J. Physiol. Regul. Integr. Comp. Physiol. 289, 663-669(2005).

3) Day, C. Metabolic syndrome, or What you will: definitions and epidemiology. Diab Vasc Dis Res 4, 32-38 (2007).

4) Dubick, M. A. Historical perspectives on the use of herbal preparations to promote health. J. Nutr. 116, 1348-1354 (1986).

5) Jung, M.; Park, M.; Lee, M. P.; Kang, Y-H.; Kang, E. S.; Kim, S. K. Antidiabetic agents from medicinal plants. Curr. Medcinal Chem. 13, 1203-1218(2006).

6) Yin, J. Y.; Zhang, H.; Ye, J. Traditional chinese medicine in treatment of metabolic syndrome. Endo. Metab. Immune. Disord. Drug Targets. 8, 99-111(2008).

7) Hasani-Ranjibar, S.; Nayebi, N.; Larijani, B., Abbdollahi, M. A systematic review of the efficacy and safety of herbal medicines used in the treatment of obesity. World Gastrenterol. 15, 3072-3085 (2009).

8) Ames, B. M.; Shigenaga, M. K.; Hagen, T. M. Oxidants, antioxidants and the degenerative diseases of aging. Proc. Natl. Acad. Sci. USA. 90, 7915-7922 (1993).

9) Ververidis, F.; Trantas, E.; Douglas, C.; Vollmen, C.; Kretzschman, G.; Panopoulas, N. Biotchnology of flavonoids and other phenylpropanoid-derived natural products. Part I: Chemical diversity, impact on plant biology and human health. Biotechnol. J. 2, 12141234 (2007).

10) Serrano, J.; Puupponen-Pimia, R.; Dauer, A.; Aura, A-M.; Saura-Calixto, F. Tannins: Current knowledge of food sources, intake, bioavailability and biological effects. Mol. Nutr. Food Res. 53, S310-S329 (2009).

11) Blade, C.; Arola, L.; Salvado, M-J. Hypolipemic effects 
of proanthocyanidins and their underlying biochemical and molecular mechanisms. Mol. Nutr. Food Res. 54, 37-59 (2010).

12) Takeshita, M.; Ishida, Y.; Akamatsu, E.; Ohmori, Y.; Sudoh, M.; Uto. H.; Tsubouchi, H.; Kataoka, H. Proanthocyanidin from blueberry leaves suppresses expression of subgenomic hepatitis C virus RNA. J. Biol. Chem. 284, 21165-21176(2009).

13) Sakaida, H.; Nagao, K.; Higa, K.; Shirouchi, B.; Inoue, N.; Hidaka, F.; Kai, T.; Yanagita, T. Effect of vaccinium ashei reade leaves on angiotensin converting enzyme activity in vitro and on systolic blood pressure of spontaneously hypertensive rats in vivo. Biosci. Biotechnol. Biochem. 71, 2335-2337 (2007).

14) Shaughnessy, K. S.; Boswall, J. A.; Scanlan, A. P.; Gottschall-Pass, K. T.; Sweebey, M. I. Diets containing blueberry extract lower blood pressure in spontaneously hypertensive stroke-prone rats. Nutr. Res. 29, 130-138 (2009).

15) Basu, A.; Du, M.; Leyva, M. J.; Sanchez, K.; Betts, N. M.; Wu, M.; Aston, C. E,; Lyons, T. J. Blueberries decrease cardiovascular risk factors in obese men and women with metabolic syndrome. J. Nutr. 140, 1582-1587 (2010).

16) Cignarella, A.; Nastasi, M.; Cavalli, E.; Puglisi, L. Novel lipid-lowering properties of Vaccinium myrtillus L. leaves, a traditional antidiabetic treatment, in several models of rat dyslipidaemia: A comparison with ciprofibrate. Thromb. Res. 84, 311-322(1996).

17) Martineau, L. C.; Couture, A.; Spoor, D. Anti-diabetic properties of the Canadian lowbush blueberry Vaccinium angustifolium Ait. Phytomedicine. 13, 612-623 (2006).

18) Stull, A. J.; Cash, K. C.; Johnson, D. J.; Champagne, C. M.; Cefalu, W. T. Bioactive in blueberries improve insulin sensitivity in obese, insulin- resistant men and women. J. Nutr. 140, 1764-1768(2010).

19) American Institute of Nutrition. Report of the American Institute of Nutrition ad hoc committee on standards for nutritional studies. J. Nutr. 107, 1340-1348 (1977).

20) Folch, J.; Lees, M.; Sloane-Stanley, G. H. A simple method for the isolation and purification of total lipides from animal tissues. J. Biol. Chem. 226, 497-509 (1957).

21) Senanayake, G. V. K.; Maruyama, M.; Shibuya, K.; Sakono, M.; Fukuda, N.; Morishita, T.; Yukizaki, C.; Kawano, M.; Ohta, H. The effects of bitter melon (Momordica charantia) on serum and liver triglyceride levels in rats. J. Ethnopharmacol. 91, 257-261 (2004).

22) Nagao, K.; Higa, K.; Shirouchi, B.; Nomura, S.; Inoue, N.; Inafuku, M.; Yanagita, T. Effect of vaccinium ashei reade leaves on lipid metabolism in otsuka long-evans tokushima fatty rats. Biosci. Biotechnol. Biochem. 72, 1619-1622 (2008).

23) Kim, H.; Bartley, G.; Rimando, A. M.; Yokoyama, W. Hepatic gene expression related to lower plasma cholesterol in hamsters fed high-fat diets supplemented with blueberry peels and peel extract. J. Agri. Food Chem. 58, 3984-3991 (2010).

24) Kalt, W.; Foote, K.; Fillmore, S. A. E.; Lyon, M.; Van, Lunen, T. A.; McRae, K. B. Effect of blueberry feeding on plasma lipids in pigs. Bri. J. Nutr. 100, 70-78 (2008).

25) Wu, X.; Kang, J.; Xie, C.; Burris, R.; Ferguson, M. E.; Badger, T. M.; Nagarajan, S. Dietary blueberries attenuate atherosclerosis in apolipoprotein E-deficient mice by upregulating antioxidant enzyme expression. $J$ Nutr. 140, 1628-1632(2010).

26) Fungwe, T. V.; Cagen, L. M.; Cook, G. A.; Wilcox, H. G.; Heimberg, M. Dietary cholesterol stimulates hepatic biosynthesis of triglyceride and reduces oxidation of fatty acids in the rat. J. Lipid Res. 34, 933-941 (1993).

27) Fungwe, T. V.; Fox, J. E.; Cagen, L. M.; Wilcox, H. G.; Heimberg, M. Stimulation of fatty acid biosynthesis by dietary cholesterol and of cholesterol synthesis by dietary fatty acid. J. Lipid Res. 35, 311-318(1994).

28) Inoue, N.; Nagao, K.; Nomura, S.; Shirouchi, B.; Inafuku, M.; Hirabaru, H.; Nakahara, N.; Nishizono, S.; Tanaka, T.; Yanagita, T. Effect of Vaccinium ashei Reade leaf extracts on lipid metabolism in obese OLETF rats. Biosci. Biotechnol. Biochem. 75, 23042308(2011).

29) Liang, Y.; Chen, J.; Zuo, Y.; Ma, K-Y.; Jiang Y.; Huang, Y.; Chen, Z-Y.: Blueberry anthocyanins at doses of $0.5 \%$ and $1 \%$ lowered plasma cholesterol by increasing fecal excretion of acidic and neutral sterols in hamsters fed a cholesterol-enriched diet. Eur. J. Nutr. Published online: 09 June (2012).

30) Sotillo, D. R.; Hadley, M. Chlorogenic acid modifies plasma and liver concentrations of cholesterol, triacylglycerol, and minerals in (fa/fa) Zucker rats. J. Nutr. Biochem. 13, 717-726 (2002).

31) Prosky, L.; Asp, N. G.; Schwizer, T. F.; DeVries, J. W.; Furda, I.: Determination of insoluble, soluble, and total dietary fiber in foods and food products, interlaboratory study. J. Assoc. Off. Anal. Chem. 71, 1017-1023 (1988). 